\title{
SISTEMA UNIVERSITARIO DE CALIDAD
}

\author{
University quality system
}

\author{
Diana Esmeralda Andamayo Flores ${ }^{1}$ \\ Universidad Roosevelt, Perú \\ José Manuel Castillo Custodio ${ }^{2}$ \\ Universidad Peruana los Andes, Perú
}

${ }^{1}$ Investigadora reconocida por CONCYTEC - RENACYT María Rostworowski
- Nivel I. Química Farmacéutica de profesión, Magister en Administración de los
Servicios de Salud, Doctora en Farmacia y Bioquímica y Doctora en Educación,
Directora del Instituto de Investigación de la Universidad Privada de Huancayo
Franklin Roosevelt, Presidenta del Comité de Licenciamiento de Condiciones
Básicas de Calidad, Docente Ordinaria Asociada de la Universidad Privada de
Huancayo Franklin Roosevelt, Docente que realiza investigación en la
Universidad Privada de Huancayo Franklin Roosevelt, Docente de posgrado de
la Universidad Peruana Los Andes, Evaluadora de SINEACE, Directora de la
Escuela Profesional de Ciencias Farmacéuticas y Bioquímica del 2010 al 2020,
Pas Decana del Colegio Químico Farmacéutico Departamental de Junín, Pas
tesorera del Consejo de Decanos de los Colegios Profesionales, Directora Técnica
de establecimientos farmacéuticos del 2000 al 2015, Presidenta de la Comisión
de Admisión de la Universidad Privada de Huancayo Franklin Roosevelt en el
2011, Autora de libros relacionados a las ciencias farmacéuticas y educativas.
Correspondencia a dandamayo@uroosevelt.edu.pe

${ }^{2}$ Título de Especialista en Didáctica Universitaria con mención en Ciencias de la Salud, Maestro en Administración, Doctor en Educación, Docente cesante en la categoría de Principal de la Universidad San Luis Gonzaga de Ica, Docente Principal de la Universidad Peruana Los Andes, Docente que realiza investigación en la Universidad Peruana Los Andes, Rector de la Universidad Peruana Los Andes del 2011 al 2016 y Del 2016 al 2020, Responsable de la Comisión de Alto Nivel para establecer e Implementar el Proceso de Licenciamiento de la Universidad Peruana Los Andes del 2015 al 2020, Visitante Distinguido de la Región La Libertad, Vicerrector Académico Titular de la Universidad Peruana Los Andes del 2006 al 2011, Presidente de la Comisión de Rectores en Calidad de Informantes para la Universidad Inca Garcilaso de la Vega en el 2014, Decano Encargado de la Facultad de Ciencias de la Salud en el 2006, Autor de libros relacionados a la educación. Correspondencia a jomacascus@gmail.com 


\section{RESUMEN}

El presente trabajo tiene como objetivo analizar y describir las condiciones básicas que debe cumplir el sistema universitario para que sea de calidad y pueda ofrecer a los futuros profesionales una formación idónea, pertinente que asegure que el profesional que egrese de una universidad se inserte al campo laboral con facilidad y demuestre las competencias profesionales de acuerdo al perfil profesional de las diferentes escuelas profesionales.

En un entorno caracterizado por el intenso proceso de globalización, donde los niveles de competitividad son cada vez más fuertes y determinantes en el mercado y el incesante avance de la ciencia y tecnología, han permitido una tendencia creciente y de gran importancia para el desarrollo de la humanidad, cuyos procesos tuvieron como base fundamental la acumulación de mayores y mejores niveles de conocimientos de calidad, los mismos que vienen permitiendo el análisis y solución de diversos problemas sociales, económicos, institucionales y ambientales que aún aquejan a las sociedades del mundo.

La educación superior universitaria en nuestro país, vienen enfrentando un conjunto de problemas y desafíos; donde la educación no ha permitido formar ciudadanos con las suficientes capacidades para el ejercicio profesional competente y la producción de conocimiento con alto valor social, además que no es un medio para la inclusión y movilidad social de los jóvenes de nuestro país. En ese sentido la política de aseguramiento de la calidad de la educación superior universitaria y el modelo de licenciamiento institucional de las universidades permitirán la mejora de la formación y la calidad académica, considerando como factores fundamentales que debemos tener en consideración para la contribución al desarrollo nacional; impulsando una visión de futuro, que asegure un sistema universitario de calidad, donde participen activamente el estado, la universidad, la empresa, la cooperación internacional y la sociedad en general con el 
propósito de garantizar a los jóvenes para que accedan a un servicio de educación superior de calidad con una formación integral, continua y competente.

\section{SISTEMAS Y SISTEMATIZACIÓN}

Según Suarez (2012) entendemos por sistema a un conjunto de elementos interrelacionados y organizados de acuerdo con ciertas necesidades, para el logro de un fin. Donde se afirma que un sistema no se define solo por los elementos que lo integran sino también por la organización que hace posible su funcionamiento.

En todo sistema hay elementos esenciales y accidentales, son esenciales aquéllos sin los cuales el sistema no funciona; son accidentales aquéllos que permiten al sistema funcionar mejor.

La organización debe ser flexible, especialmente en esta época de cambio, con el fin de facilitar las reformas necesarias con mayor certeza; la planificación es una acción continua sujeta a permanente crítica, a través de la información de retorno. La planificación tendrá en cuenta las posibilidades sin perder de vista las necesidades, sin hipotecar el futuro; se tendrá en cuenta el aspecto cuantitativo (número) y también el cualitativo (eficiencia y características de la enseñanza.

Para sistematizar una acción se debe considerar lo siguiente:

- Identificación de problemas y necesidades.

- Determinación de metas y objetivos.

- Proposición y selección de opciones.

- Selección de estrategias.

- Realización de la acción planeada.

- Control y evaluación.

- Revisión del sistema. 


\section{LA SISTEMATIZACIÓN DEL PROCESO DE ENSEÑANZA - APRENDIZAJE}

Al referirse a la sistematización de la educación se debe tener en cuenta que el futuro se construye y la meta se logra recorriendo un camino. Por lo tanto, el acto educativo en el sistema universitario debe planificarse teniendo en cuenta un proyecto, mediante una visión clara del camino por recorrer y teniendo en cuenta objetivos definidos.

El sistema educativo debe situarse dentro de los sistemas político, económico. Donde la educación se dirige a la vida concreta y muchos problemas educativos son muchas veces ininteligibles e inexplicables sino se hace referencia a un sistema más vasto, por ejemplo, el rendimiento académico, por consiguiente, la falta de una planificación social y económica limita las posibilidades de una planificación educativa.

Luego de un estudio de necesidades e identificación de problemas, el planificador de la educación debe tomar una opción sobre los objetivos de la educación y su metodología, teniendo en cuenta diversos escenarios como el ambiente ecológico -social, y dentro de los objetivos globales del hombre y su sociedad: culturales y políticos. Estos propósitos debidamente organizados por orden de prioridad y articulados dentro de un conjunto, formarán la llamada política educativa, donde al democratizar esta política se buscarán medios, estrategias para ponerla en práctica, estableciendo mecanismos para la implementación y decisiones que se tomarán en diversas situaciones. (Suarez, 2012).

\section{BASES LEGALES VIGENTES DE LA POLÍTICA EDUCATIVA PERUANA}

Según la Constitución política del Perú (2019). Referente al derecho a la educación del ciudadano en los siguientes artículos precisa lo siguiente: 
En el Artículo $16^{\circ}$, segundo párrafo, señala que el Estado coordina la política educativa; formula los lineamientos generales de los planes de estudios, así como los requisitos mínimos de la organización de los centros educativos; y, supervisa su cumplimiento y la calidad de la educación.

En el Artículo $18^{\circ}$, precisa que la "educación universitaria tiene como fines la formación profesional, la difusión cultural, la creación intelectual, artística y la investigación científica y tecnológica...".

"La Universidad es la comunidad de profesores, estudiantes y graduados". "Cada universidad es autónoma en su régimen normativo, de gobierno, académico, administrativo y económico. Las universidades se rigen por sus propios estatutos..."; a partir de dichos preceptos constitucionales se puede establecer que las universidades en conjunto tienen un rol y una responsabilidad con el desarrollo de nuestra sociedad, quienes deben de proveer profesionales, cultura, desarrollo intelectual, ciencia y tecnología acorde a las necesidades reales de nuestra población.

De acuerdo a la (Ley N 28044 - Ley General de Educación (28.07.2003) en los artículos siguientes detalla el concepto de educación, principios, calidad de la educación.

Artículo $2^{\circ}$.- Concepto de la educación.

La educación es un proceso de aprendizaje y enseñanza que se desarrolla a lo largo de toda la vida y contribuye a la formación integral de las personas, al pleno desarrollo de sus potencialidades, a la creación de cultura, y al desarrollo de la familia y de la comunidad nacional, latinoamericana y mundial.

Artículo $8^{\circ}$.- Principios de la educación.

La educación peruana tiene a la persona como centro y agente fundamental del proceso educativo. Se sustenta en los siguientes 
principios: La ética, la equidad, la calidad, la democracia, la interculturalidad y la conciencia ambiental.

Artículo $13^{\circ}$.- Calidad de la educación.

Es el nivel óptimo de formación que deben de alcanzar las personas para enfrentar los retos del desarrollo humano, ejercer su ciudadanía y continuar aprendiendo durante toda la vida.

Los factores que interactúan para el logro de la calidad son: Lineamientos del proceso educativo, currículos básicos, inversión mínima por estudiante, idoneidad de los docentes, carrera administrativa, infraestructura, equipamiento, servicios, materiales, investigación, innovación, organización institucional y relaciones humanas.

Artículo $29^{\circ}$.- Etapas del Sistema educativo.

Educación Superior, está destinada a la investigación, creación y difusión de conocimientos; a la proyección a la comunidad; al logro de competencias profesionales de alto nivel, de acuerdo con la demanda y la necesidad del desarrollo sostenible del país.

En la nueva ley universitaria - Ley $\mathrm{N}^{\circ} 30220$ (2014) precisa en sus articulados el marco legal que rige para el proceso de licenciamiento en el sistema universitario peruano.

En el Artículo $28^{\circ}$, Licenciamiento de universidades, se señala que las condiciones básicas que establezca la Superintendencia Nacional de Educación Superior Universitaria (SUNEDU) para el licenciamiento, están referidas como mínimo a los siguientes aspectos:

28.1 La existencia de objetivos académicos; grados y títulos a otorgar y planes de estudio correspondientes.

28.2 Previsión económica y financiera de la universidad a crearse compatible con los fines propuestos en sus instrumentos de planeamiento. 
28.3 Infraestructura y equipamiento adecuados al cumplimiento de sus funciones (bibliotecas, laboratorios, entre otros).

28.4 Líneas de investigación a ser desarrolladas.

28.5 Verificación de la disponibilidad de personal docente calificado con no menos del $25 \%$ de docentes a tiempo completo.

28.6 Verificación de los servicios educacionales complementarios básicos (servicio médico, social, psicopedagógico, deportivo, entre otros).

28.7 Existencia de mecanismos de mediación e inserción laboral (bolsa de trabajo u otros).

En la Décima Primera Disposición Complementaria Transitoria, Implementación Progresiva, se precisa que la Superintendencia Nacional de Educación Superior Universitaria (SUNEDU) aprobará un plan de Implementación progresiva, lo que implica inicialmente, la constatación de las condiciones básicas de calidad en las universidades con autorización provisional. Las universidades autorizadas deberán adecuarse a las condiciones básicas de calidad en el plazo que la Superintendencia Nacional de Educación Superior Universitaria (SUNEDU) establezca, sometiéndose a la supervisión posterior.

El D.S. N $^{\circ}$ 016-2015-MINEDU, aprueba la Política de Aseguramiento de la Calidad de la Educación Superior Universitaria (2015).

La Política de Aseguramiento de la Calidad de la Educación Superior Universitaria, ha sido formulada principalmente considerando los alcances de la Constitución Política del Perú; las referencia de la Décima Segunda Política de Estado del Acuerdo Nacional; el Artículo $4^{\circ}$ de la Ley Orgánica del Ministerio de Educación - Decreto Ley $\mathrm{N}^{\circ}$ 25762; Proyecto Educativo Nacional al 2021, Objetivo Estratégico 5: Educación Superior de Calidad se 
convierte en factor favorable para el desarrollo y competitividad nacional; la Ley $\mathrm{N}^{\circ} 30220$, en el Artículo $1^{\circ}$, establece que el Ministerio de Educación es el ente rector de la política de aseguramiento de la calidad de la educación superior universitaria entre otros aspectos.

La política referida es de aplicación de todos los actores involucrados en el Sistema de Educación Superior Universitario Peruano; cuyo objetivo es garantizar a todos los jóvenes del País tengan la oportunidad de acceder a un servicio educativo universitario de calidad, que ofrezca una formación integral y de perfeccionamiento continuo, centrado en el logro de un desempeño profesional competente y en la incorporación de valores ciudadanos que permiten una reflexión académica del país a través de la investigación.

Los factores de la Política de Aseguramiento de la Calidad de la Educación Superior Universitaria, hace referencia a los aspectos siguientes:

- Introducción.

- Fundamento y contexto de la Política de Aseguramiento de la Calidad de la Educación Superior Universitaria.

- La Visión: Hacia un Sistema Universitario de Calidad.

- Alcance de la Política.

- Objetivo General de la Política.

- Principios de la Política Pilares de la reforma para la construcción de un Sistema de Aseguramiento de la Calidad.

- Lineamientos y Acciones Estratégicas de la Política.

POLÍTICA DE ASEGURAMIENTO DE LA CALIDAD Y MODELO DE LICENCIAMIENTO INSTITUCIONAL

En el D.S. $\mathrm{N}^{\circ} 016$ - 2015 - MINEDU, Política de Aseguramiento de la Calidad de la Educación Superior Universitaria 
(2015). Precisa aspectos que son esenciales para su comprensión como: El fundamento y contexto de la política, la visión hacia un sistema de calidad, el alcance de la política, objetivo general de la política, los principios de la política, y fundamentalmente como parte medular señala los pilares de la reforma para la construcción del sistema de calidad, precisa cuatro pilares, 11 lineamientos con los cuales se pretende asegurar un Sistema de Educación Superior Universitaria de Calidad. (Figura 1).

\section{Figura 1.}

Política de Aseguramiento de la Calidad de la Educación Superior Universitaria.
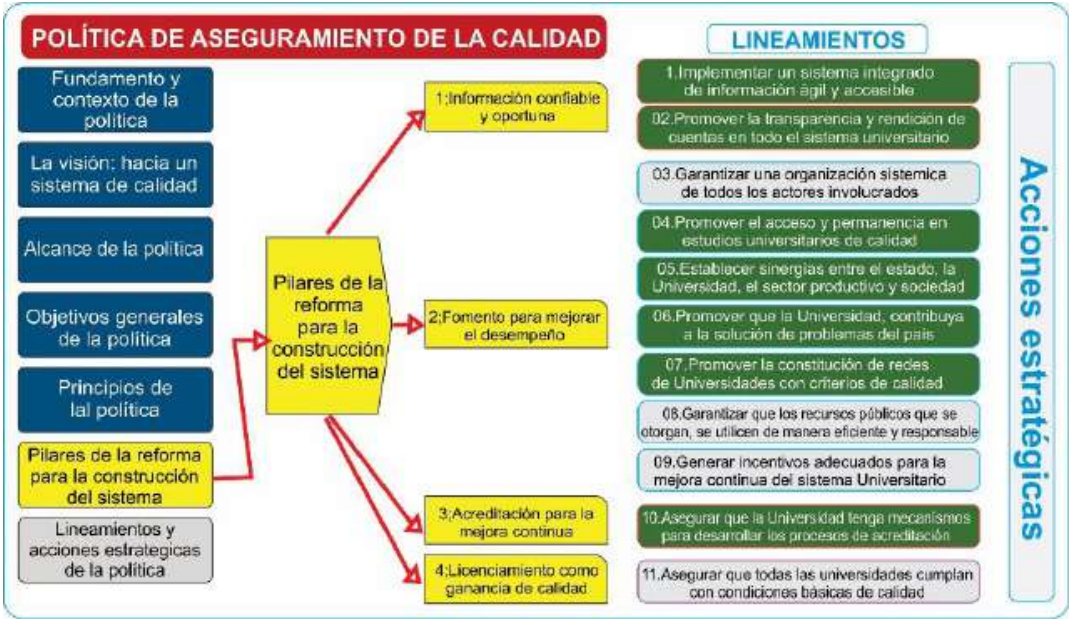

Nota. Elaboración propia en base al Decreto Supremo $N^{\circ} 016-2015$ MINEDU.

La fundamentación de la política a manera de síntesis para una mejor comprensión se detalla a continuación:

\section{FUNDAMENTO Y CONTEXTO:}

La Política de Aseguramiento de la Calidad de la Educación Superior Universitaria, ha planteado como principal objetivo, asegurar a todos los jóvenes del país tener la oportunidad de acceder a una 


\section{Diana Esmeralda Andamayo}

\section{José Manuel Castillo Custodio}

educación universitaria de calidad que permita su realización personal, y los forme como ciudadanos y profesionales de alta productividad.

Dicha política se ha fundamentado esencialmente en el proceso de crecimiento de nuestra economía, la mejora relativa de la calidad de vida de la población, desarrollo de las ciudades más importantes y el optimismo por el futuro.

Considerando que el Estado tiene la delicada responsabilidad de promover e impulsar el desarrollo del País, por lo que debemos de precisar que la Educación es un factor fundamental para el crecimiento y desarrollo, adaptándonos a los entornos cambiantes y dinámicos propios de la modernidad.

Por ello, la universidad es un componente estratégico para articular la educación, con el mercado laboral y los sectores productivos; donde el Estado debe de garantizar el derecho a la educación de calidad, asegurando niveles óptimos en el servicio educativo superior universitario el cual tendrá una incidencia directa en el proceso de crecimiento y desarrollo sostenible de nuestro país.

En ese sentido, se debe comprender que la calidad universitaria está relacionada con las tareas de la docencia, las actividades de investigación, su propia gestión, administración y organización, el uso de sus recursos, su política de bienestar institucional y sus formas de articulación con los actores de su entorno.

Las universidades a nivel de América Latina, han experimentado un proceso de expansión de matrícula en el ámbito universitario, a nivel de nuestro país entre 1990 y el 2012 se registró una tasa de crecimiento de matrícula promedio $2.5 \%$ anual, con una débil o nula planificación de la misma y una ausencia de políticas de aseguramiento de la calidad, por lo que se aseveraba la existencia de una educación superior universitaria sin capacidad de formar ciudadanos con altas capacidades para el ejercicio profesional competente y la generación de conocimiento con alto valor social; y 
no se constituía en un medio para la inclusión y movilización social de los jóvenes del país.

\section{VISIÓN DE FUTURO: HACIA UN SISTEMA UNIVERSITARIO DE CALIDAD}

El país requiere que la educación superior universitaria se organice y opere de manera sistémica, según las exigencias de calidad, donde el Estado es garante de la educación de la calidad y la universidad es el proveedor del servicio de calidad y la sociedad asume el rol de vigilancia académica e institucional.

La Universidad, provee el servicio educativo universitario valorando los factores como: Los estudiantes, docentes, gestión universitaria, disciplinas y programas profesionales, investigación e infraestructura.

\section{OBJETIVO DE LA POLÍTICA}

Garantizar que todos los jóvenes del país tengan la oportunidad de acceder a un servicio educativo de calidad, centrado en el logro de un desempeño profesional competente y la incorporación de valores ciudadanos.

\section{PRINCIPIOS DE LA POLÍTICA (FIGURA 2)}

- Autonomía y rectoría responsables.

- El estudiante como centro.

- Inclusión y equidad.

- Calidad y excelencia académica.

- Desarrollo del país. 
Figura 2.

Principios de la Política de Aseguramiento de la Calidad de la Educación Superior Universitaria.

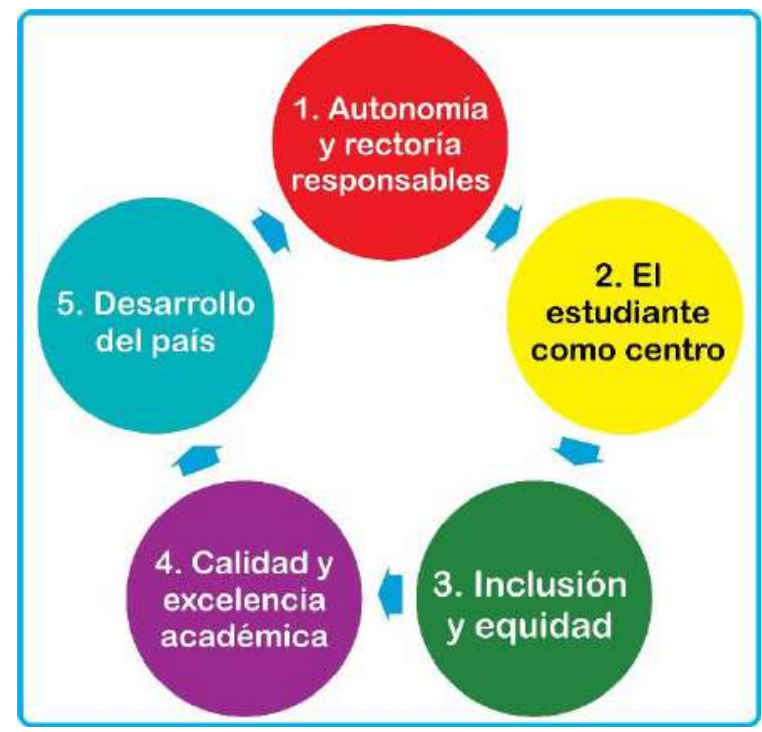

Nota. Elaboración propia en base al Decreto Supremo $N^{\circ}$ 016-2015MINEDU.

\section{PILARES Y LINEAMIENTOS DE LA REFORMA}

\section{Información confiable y oportuna.}

- Implementar un sistema integrado de información ágil y accesible de la educación superior universitaria que permita la mejor toma de decisiones para el Estado y sociedad.

- Promover la transparencia y rendición de cuentas en todo el Sistema Universitario, así como la continua vigilancia académica e institucional por parte de la sociedad.

\section{Fomento para mejorar el desempeño.}

- Garantizar una organización sistémica de todos los actores involucrados en la Educación Superior Universitaria, donde estos 
conocen y ejercen sus roles responsablemente en un marco conceptual, programático y normativo, ordenado y supervisado.

- Promover el acceso y permanencia en estudios universitarios de pregrado y posgrado de calidad, hasta su culminación, sin ningún tipo de distinción.

- Establecer sinergias entre el Estado, la universidad, el sector productivo y la sociedad civil organizada, así como mecanismos de concertación para lograr una formación integral, tanto profesional como en valores, que aporte al desarrollo del país.

- Promover que la universidad, como institución social y académica, contribuya a la solución de los problemas del país, a través de la investigación, en el marco de una sociedad igualitaria, inclusiva y democrática.

- Promover la constitución de redes de universidades con criterios de calidad, pertinencia, responsabilidad social y la internacionalización.

- Garantizar que los recursos públicos que se le otorgan a la universidad se utilicen de manera eficiente y con responsabilidad para lograr una mejora en la prestación del servicio educativo superior universitario.

- Generar incentivos adecuados para la mejora continua del Sistema Universitario.

\section{Acreditación para la mejora continua.}

- Asegurar que la universidad tenga mecanismos para desarrollar procesos de acreditación institucional y de sus programas académicos, en búsqueda de la excelencia del servicio educativo superior universitario. 
Licenciamiento como garantía de las condiciones básicas de calidad.

- Asegurar que todas las universidades y sus programas de estudios conducentes a grados y títulos académicos cumplan con condiciones básicas de calidad para obtener y mantener su licenciamiento.

\section{MODELO DE LICENCIAMIENTO Y SU IMPLEMENTACIÓN EN EL SISTEMA UNIVERSITARIO PERUANO}

Resolución de Consejo Directivo $\mathrm{N}^{\circ}$ 006-2015SUNEDU/CD, aprueban el "modelo de licenciamiento y su implementación en el sistema universitario peruano del 24.11.2015".

El Modelo de Licenciamiento responde a la realidad de nuestro país y al nivel alcanzado por el sistema universitario peruano; tiene como referencia a la Política de Aseguramiento de la Calidad de la Educación Superior Universitaria y experiencias desarrolladas a nivel internacional, considera los aspectos siguientes:

\section{OBJETIVO DEL LICENCIAMIENTO}

El licenciamiento tiene como objetivo general, verificar que las universidades cumplan con las Condiciones Básicas de Calidad (CBC) para ofrecer el servicio educativo superior universitario y alcancen la licencia que las habilite a prestar el servicio educativo.

La verificación de las Condiciones Básicas de Calidad (CBC), supone alcanzar los objetivos específicos siguientes:

- Proteger a los usuarios del servicio, brindándoles información confiable y útil para la toma de decisiones.

- Contribuir en la generación y desarrollo del sistema de información, que ayude a las universidades en sus planes y al MINEDU en la formulación de políticas públicas. 
- Asegurar la capacidad de las universidades para desarrollar nuevos programas educativos de calidad.

- Promover la eficacia, eficiencia e innovación en la Educación Superior Universitaria.

\section{NATURALEZA DE LAS CONDICIONES BÁSICAS DE CALIDAD}

El licenciamiento constituye un mecanismo de protección para la sociedad, al adecuar a las instituciones, filiales y programas al cumplimiento de las Condiciones Básicas de Calidad (CBC), opera como un primer nivel que las universidades deben de cumplir. (Tabla $1)$.

\section{Tabla 1.}

Condiciones Básicas de Calidad y Número de Indicadores.

\begin{tabular}{|c|c|c|}
\hline & Condiciones & $\begin{array}{c}\mathbf{N}^{\circ} \text { de } \\
\text { indicadores }\end{array}$ \\
\hline 1) & $\begin{array}{l}\text { Existencia de objetivos académicos, grados y } \\
\text { títulos a otorgar y planes de estudio } \\
\text { correspondientes. }\end{array}$ & 8 \\
\hline 2) & $\begin{array}{l}\text { Oferta educativa a crearse compatible con los fines } \\
\text { propuestos en los instrumentos de planeamiento. }\end{array}$ & 7 \\
\hline 3) & $\begin{array}{l}\text { Infraestructura y equipamiento adecuados al } \\
\text { cumplimiento de sus funciones (aulas, bibliotecas, } \\
\text { laboratorios, entre otros). }\end{array}$ & 15 \\
\hline 4) & Líneas de investigación a ser desarrolladas. & 8 \\
\hline 5) & $\begin{array}{l}\text { Verificación de la disponibilidad de personal } \\
\text { docente calificado con no menos del } 25 \% \text { de } \\
\text { docentes a tiempo completo. }\end{array}$ & 4 \\
\hline 6) & $\begin{array}{l}\text { Verificación de los servicios educacionales } \\
\text { complementarios básicos (Servicio médico, } \\
\text { social, psicopedagógico, deportivo, entre otros). }\end{array}$ & 8 \\
\hline 7) & $\begin{array}{l}\text { Existencia de mecanismos de mediación e } \\
\text { inserción laboral (Bolsa de trabajo u otros) }\end{array}$ & 4 \\
\hline 8) & Transparencia de universidades. & 1 \\
\hline & TOTAL: & 55 \\
\hline
\end{tabular}




\section{CARÁCTER OBLIGATORIO DEL PROCESO DE LICENCIAMIENTO}

De acuerdo al artículo $13^{\circ}$ de la Ley Universitaria (2014), el licenciamiento es una condición obligatoria para ofrecer el servicio educativo superior universitario. La obligatoriedad del proceso de licenciamiento se sustenta en la necesidad que las universidades operen dentro de un marco de aseguramiento de la calidad.

\section{ENFOQUE INSTITUCIONAL DEL MODELO DE LICENCIAMIENTO}

Se inicia el proceso con un enfoque institucional, verificando el cumplimiento de la totalidad de las Condiciones Básicas de Calidad (CBC) en la universidad y sus filiales.

A medida que la Superintendencia Nacional de Educación Superior Universitaria (SUNEDU) evalué a las universidades del sistema universitario, dentro del marco del Licenciamiento de Programas con el fin de aprobar o denegar las solicitudes de licenciamiento de programas conducentes a grado académico o título. (Figura 3).

Figura 3.

Modelo de Licenciamiento Institucional.

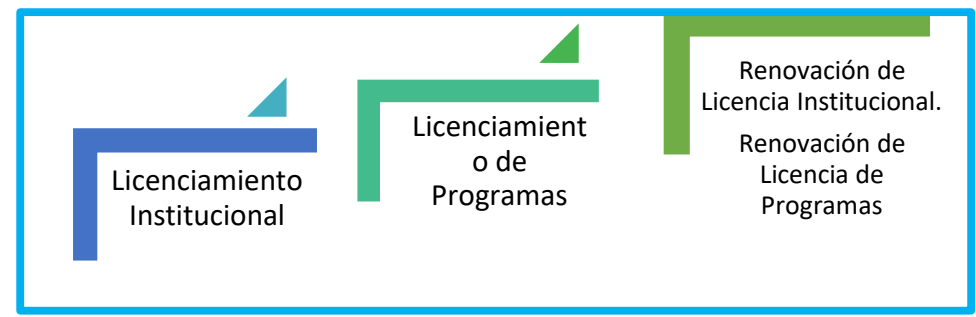

Nota: Resolución de Consejo Directivo N 006-2015-SUNEDU/CD. 


\section{ELEMENTOS DEL CURRÍCULO PARA UNA FORMACIÓN DE CALIDAD}

El sistema universitario de calidad, para garantizar la formación profesional de los estudiantes debe de preocuparse en diseñar un currículo pertinente donde debe tener en cuenta los elementos que conforman el currículo.

Sovero (2008). Refiere en los elementos del currículo del sistema nacional que muchos autores reconocen que el currículo comprende una serie elementos básicos. Estos elementos son trabajados sobre todo por la concepción sistemática del currículo y pretenden dar respuesta, de alguna manera, a una serie de interrogantes sobre la finalidad, los sujetos, los medios, entre otros.

Así surgen los perfiles, competencias, contenidos, estrategias y evaluación. Cada uno de estos elementos del currículo, se plantean como respuesta a las necesidades de un país.

- Los perfiles describen las características de los sujetos involucrados en las acciones educativas tanto al principio como al final de dichas acciones. Los perfiles que describen las características deseables del estudiante al terminar un proceso de formación cumplen una función orientadora en la propuesta curricular. En nuestro medio adquieren diferentes denominaciones: perfil del egresado, perfil ideal, perfil básico, etc.

- Las competencias nos señalan el para qué de la acción educativa y buscan satisfacer las necesidades básicas de aprendizaje de un ser humano, que les permita demostrar el saber, saber hacer y el saber ser.

Se puede definir también como la capacidad para realizar un desempeño eficiente y eficaz depende de la integración de tres elementos: la información, los procedimientos y las actitudes, denominados a estos elementos, respectivamente, componente 


\section{Diana Esmeralda Andamayo}

\section{José Manuel Castillo Custodio}

conceptual, componente procedimental y componente actitudinal. Se trata de tres aspectos de órdenes distintas:

- Componente conceptual, es el conjunto organizado de conceptos, definiciones y datos de tipo teórico.

- Componente procedimental, es el manejo de técnicas, métodos y estrategias que se aplican hacia una meta y cuyas acciones acontecen en su orden o sucesiones lógicas.

- Componente actitudinal, es la disposición del individuo ante diversas situaciones, objetos, valores e interés, que se visualiza en actitudes favorables.

- Los contenidos responden ¿Qué se aprende? Actualmente son concebidos como elementos útiles para la construcción reflexiva del conocimiento y de las estructuras significativas en los estudiantes. Por lo general se organizan en áreas, niveles, ciclos, cursos o temas, atendiendo a criterios psicológicos, lógicos y sociales.

- Las estrategias responden a la pregunta ¿Cómo se aprende? Determinan el tipo de experiencias, condiciones e interacciones que se van a generar con la finalidad de favorecer el aprendizaje significativo en el alumno. En este sentido, actualmente se sostiene que las estrategias para orientar el aprendizaje deben ofrecer escenarios contextualizados y apoyados en las concepciones previas de los estudiantes. Son de naturaleza dinámica y variada.

- La evaluación, es un elemento que sirve para valorar el aprendizaje del estudiante y para tomar decisiones sobre el currículo. Pretende ser un proceso cualitativo, formativo participativo y puede ser efectuado desde un enfoque de investigación-acción.

La evaluación está inexorablemente unida a la calidad de forma inseparable en el comportamiento y los juicios de valor de las personas. Los agentes de cambio educativo podrán encontrar en las 
respuestas de por qué se puede o no introducir los nuevos conceptos de calidad en la educación. (Buendía y López, 1999).

Por lo tanto se considera necesario y obligatorio que las universidades del Perú deben tener el licenciamiento institucional para posteriormente realizar la acreditación, de la evaluación y regulación de la calidad que están relacionadas en sus propósitos y procedimientos, que es imposible considerarlas separadamente se acredita conforme a un proceso de evaluación y de seguimiento, para disponer de información fidedigna y objetiva sobre la calidad de las instituciones y programas universitarios, sea que estén en su fase de reconocimiento inicial o en pleno desarrollo de su modelo educativo (Becerra, 2014).

La calidad en educación superior, que las instituciones lo vienen manejando de acuerdo a sus planificaciones, se tiene que en el país se está convirtiendo en la actualidad como una política pública, donde el estado debe preocuparse y debe considerar dentro del presupuesto nacional partidas que estén destinadas para este fin. Teniendo algunas instituciones de educación superior privadas y estatales la preocupación de acreditar algunos programas de estudio bajo el modelo de acreditación de SINEACE. Considerando que el tema de la calidad de la educación no puede plantearse al margen del Proyecto País, quiere decir y tal como refiere Yamada et al. (2013) debe estar sujeto al contexto en el que se van a desarrollar los modelos de acreditación porque será este contexto en el que cobren sentido. En palabras de Gustavo Yamada debe haber "mayor conciencia de ajustar los sistemas nacionales de aseguramiento de la calidad a las necesidades y realidades de cada país". Si el contexto cambia, las instituciones también y por lo tanto los modelos de acreditación y el planteamiento de estándares que midan dicha calidad deberán ser modificados de acuerdo a estos cambios. El concepto de "educación de calidad", tal como defiende la UNESCO, se plantea así como un 
concepto dinámico que evoluciona en tanto evoluciona el concepto mismo de educación.

(...) creemos que si hay algo clave que se debe exigir en todo proceso de evaluación de la calidad y pertinencia de las instituciones de educación superior es su vinculación con las necesidades reales de la economía y la sociedad. Yamada et al. (2013).

\section{CONCEPCIÓN DEL NUEVO MODELO DE ACREDITACIÓN}

(...) El nuevo modelo no debe entenderse como un conjunto de ajustes, modificaciones y transformaciones en la matriz de evaluación, sino como un giro significativo en la concepción de la evaluación de la calidad educativa. Este nuevo modelo concibe la evaluación de la calidad como un proceso formativo que ofrece a las instituciones oportunidades para analizar su quehacer, introducir cambios para mejorar de manera progresiva, permanente y sostenida, fortalecer su capacidad de auto regulación e instalar una cultura de calidad institucional a través de la mejora continua. Cabe precisar, que la evaluación de la calidad es distinta de la medición: la primera se expresa en términos cualitativos a diferencia de la segunda, que se establece cuantitativamente. Una evaluación hace uso de la medición, pero va más allá, emite un juicio de valor como consecuencia del análisis profundo de los aspectos involucrados. Dicho juicio se expresa en textos, que explicitan la apreciación del par evaluador Esta nueva concepción demanda un cambio de actitudes, así como el desarrollo de capacidades tanto en evaluados como en evaluadores. (SINEACE, 2016).

\section{ACREDITACIÓN COMO PROCESO DE MEJORA CONTINUA}

(...) La acreditación muchas veces se asocia a un proceso, que tiene como objetivo cumplir los estándares o requisitos establecidos por el organismo acreditador, desligándose del fin más importante que es la mejora continua. La acreditación, entendida como reconocimiento público al cumplimiento de estándares por parte de 
una institución, por sí misma no mejora la calidad. La mejora nace y se construye al interior de las instituciones, y en ello radica el valor de la autoevaluación. En tal sentido, la autoevaluación se constituye en el mecanismo por excelencia, que permite identificar y superar brechas de calidad a partir de la elaboración e implementación de planes de mejora. La evaluación constituye la estrategia que impulsa el cambio y la mejora. Los procesos de cambio no son únicos, ni se establecen de manera general para todos, sino que responden a procesos internos impulsados en cada institución, de acuerdo a sus particularidades, a su diversidad Por su parte, la acreditación establece hitos en el camino de la mejora continua. Si bien el proceso de mejora continua, implica consideraciones técnicas y de financiamiento, demanda un compromiso sostenido de parte de los actores involucrados, y particularmente de la institución y de quienes lideran dicho proceso. (SINEACE, 2016) " Entendiendo la mejora continua como la actividad recurrente para mejorar el desempeño" (International Organization for Standardization - ISO. (2015), se debe considerar, tanto el establecer objetivos para cumplir con el perfil de egreso, como oportunidades para la mejora de los procesos. (SINEACE, 2016).

\section{ESTRUCTURA DEL MODELO}

El SINEACE ha identificado una estructura, que consta de cuatro categorías generales o dimensiones: la primera dimensión central de formación integral, la segunda dimensión de gestión estratégica, la tercera dimensión de soporte institucional; y la cuarta de resultados.

(...) en educación superior universitaria es importante que cada institución tenga en cuenta su entorno (local, nacional e internacional) para definir el perfil de egreso de los estudiantes, debiendo considerar las particularidades expresadas en la misión, visión y valores que busca desarrollar en los egresados. Es así que el perfil de egreso tiene consistencia interna y externa. (Toro, 2012) y es fundamental que se logre en el proceso de formación profesional. 
A continuación en la Figura 4 se observa las cuatro dimensiones con el marco conceptual que avala lo referido.

Figura 4.

Estructura de la matriz de estándares.
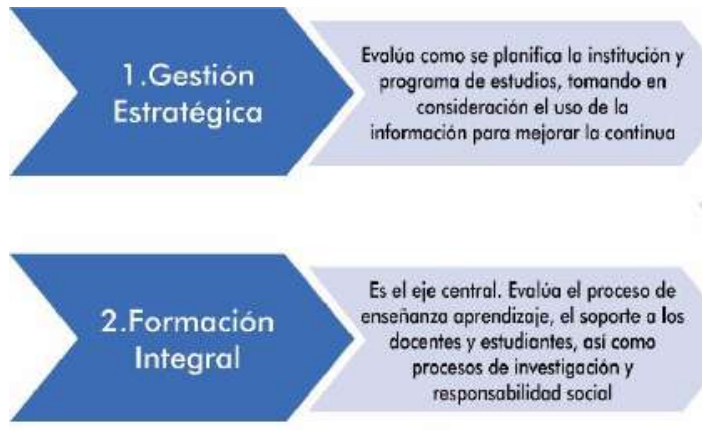

4 RESULTADOS

Verificación de resultados de aprendisaje o perfil de egreso y objefivos educacionales
3.Soporte

Institucional
Evalúa los aspectos relacionados con la gestión de recursos infraestructura y el soparte para lograr el bienestar de los miembros de la instifución educotiva.

\section{MATRIZ DE ESTÁNDARES}

El nuevo modelo de acreditación y la matriz de estándares se establece con el objetivo de llamar a la reflexión y promover el análisis y valoración de la relación entre: qué es lo que persigue el programa de estudios, y qué es lo realiza, qué logros obtiene como resultado y qué tiene que hacer para mejorar. Si encuentra debilidades. Pretende ser una herramienta de gestión que potencie la autoevaluación, instale una práctica de mejora continua y conduzca hacia la autorregulación. La matriz de evaluación vigente está estructurada en 4 dimensiones, 12 factores y 34 estándares que se acompañan de criterios a evaluar. Para evaluar el cumplimiento de cada uno de los estándares, el programa de estudios debe demostrar mediante evidencias verificables, que permita exhibir lo exigido. Las fuentes de verificación pueden ser diversas, pero en todos los casos 
sustentar el cumplimiento del estándar, teniendo en cuenta que cada institución tiene sus mecanismos y medios que les permiten el logro de los estándares, sin pretender que sea uniforme los medios de evidenciar para todas las instituciones y programas, se debe respetar la autonomía de gestión. (Tabla 2).

\section{Tabla 2.}

Matriz de estándares.

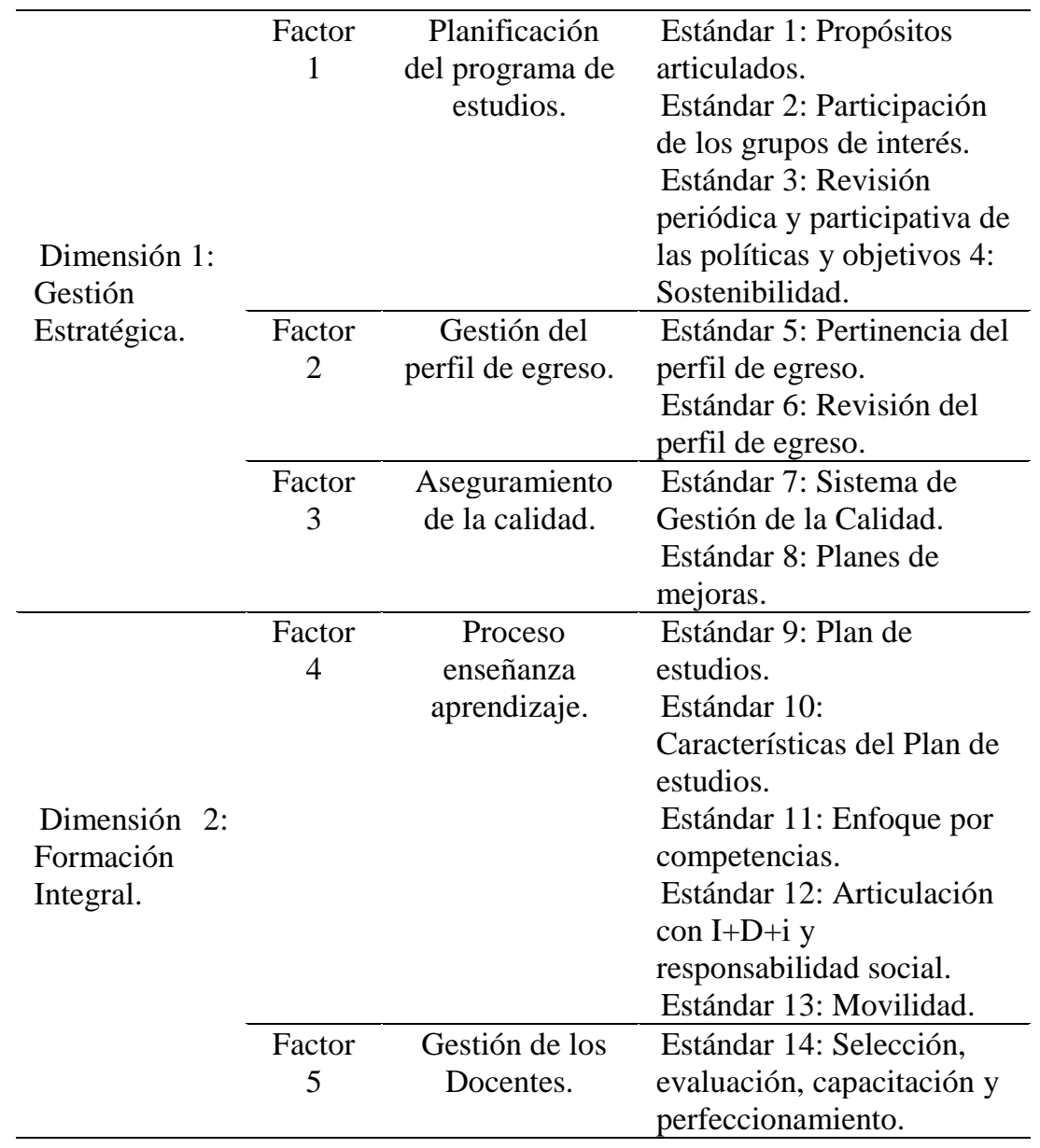


Estándar 15: Plana docente adecuada.

Estándar 16:

Reconocimiento de las actividades de labor docente.

Estándar 17: Plan de desarrollo académico del docente.

\begin{tabular}{cc}
\hline Factor & Seguimiento a \\
6 & estudiantes.
\end{tabular}
Estándar 18: Admisión al programa de estudios. Estándar 19: Nivelación de ingresantes. Estándar 20: Seguimiento al desempeño de los estudiantes. Estándar 21. Actividades extracurriculares.

\begin{tabular}{cc}
\hline Factor & Investigación, \\
7 & desarrollo \\
& tecnológico e \\
& innovación.
\end{tabular}

Estándar 22. Gestión y calidad de la I+D+i realizada por docentes. Estándar 23. I+D+i para la obtención del grado y el título.

Estándar 24. Publicaciones de los resultados de $\mathrm{I}+\mathrm{D}+\mathrm{i}$.

\begin{tabular}{|c|c|c|c|}
\hline & $\begin{array}{c}\text { Factor } \\
8\end{array}$ & $\begin{array}{l}\text { Responsabilida } \\
\text { d social } \\
\text { universitaria. }\end{array}$ & $\begin{array}{l}\text { Estándar 25: } \\
\text { Responsabilidad social. } \\
\text { Estándar 26: } \\
\text { Implementación de } \\
\text { políticas ambientales. }\end{array}$ \\
\hline & $\begin{array}{c}\text { Factor } \\
9\end{array}$ & $\begin{array}{l}\text { Servicios de } \\
\text { bienestar. }\end{array}$ & Estándar 27: Bienestar. \\
\hline $\begin{array}{l}\text { Dimensión 3: } \\
\text { Soporte } \\
\text { Institucional }\end{array}$ & $\begin{array}{c}\text { Factor } \\
10\end{array}$ & $\begin{array}{l}\text { Infraestructura } \\
\text { y soporte. }\end{array}$ & $\begin{array}{l}\text { Estándar 29: } \\
\text { Mantenimiento de la } \\
\text { infraestructura. } \\
\text { Estándar 30: Sistema de } \\
\text { Información y } \\
\text { Comunicación. }\end{array}$ \\
\hline
\end{tabular}


Estándar 31: Centros de información y referencia.

\begin{tabular}{cll}
\hline $\begin{array}{c}\text { Factor } \\
11\end{array}$ & $\begin{array}{c}\text { Recursos } \\
\text { humanos. }\end{array}$ & $\begin{array}{l}\text { Estándar 32: Recursos } \\
\text { Humanos para la Gestión } \\
\text { del programa de estudios. }\end{array}$ \\
\hline Factor & $\begin{array}{l}\text { Verificación del } \\
12\end{array}$ & $\begin{array}{l}\text { Estándar 33: Logro de } \\
\text { perfil de egreso. } \\
\end{array}$ \\
& & $\begin{array}{l}\text { Estándar 34: Seguimiento } \\
\text { a egresados y objetivos } \\
\text { educacionales. }\end{array}$ \\
\hline
\end{tabular}

Nota: Elaboración propia a partir del Modelo de Acreditación para Programas de Estudios de Educación Superior Universitaria Explicación de estándares y criterios. SINEACE. 2017.

\section{CONCLUSIONES}

El Sistema universitario, debe engranarse contemplando los aspectos políticos, económicos y sociales, para estar articulada dentro de la política educativa del país de tal manera que debe ser planificada en forma pertinente, garantizando la educación superior de calidad.

Las bases legales que sostienen la política educativa del Perú debe ser socializada y aplicada respetando los articulados que sostiene el marco educativo para que se lidere con democracia y enfocando al desarrollo del país con la preparación oportuna e idónea de sus jóvenes estudiantes quienes serán los visionarios para construir el desarrollo educativo garantizando profesionales competitivos.

La política de aseguramiento de la calidad y modelo de licenciamiento institucional se está implementando y al licenciamiento se están adecuando todas las universidades del Perú para cumplir con las ocho condiciones básicas de calidad que garantizarán la formación profesional de los estudiantes 


\section{Diana Esmeralda Andamayo

idóneamente, que les permita demostrar las competencias para el que fueron formados y puedan insertarse al campo laboral sin ningún problema. Mientras que las políticas de aseguramiento de la calidad se convierten en el principal documento orientador del proceso de reforma del ámbito universitario y de su evolución y reflexión progresiva, donde todos los actores deben involucrarse en el sistema universitario con planificación y estrategias que orienten hacia el logro de la calidad educativa.

El sistema universitario de calidad, tiene como misión la garantía de la formación profesional de sus egresados y debe preocuparse en diseñar un currículo que esté orientado al logro del perfil del egresado, el cual debe ser revisado, actualizado a las necesidades de la sociedad.

La acreditación, se entiende como un reconocimiento público al cumplimiento de estándares por parte de una institución, por sí misma no mejora la calidad. La mejora se realiza desde la parte interna de la institución, en el cual es importante ejecutar la autoevaluación y luego plantearse planes de mejora que contribuya al logro de los estándares alineados al perfil del egresado. El nuevo modelo de acreditación está conformado por cuatro dimensiones, doce factores y treinta y cuatro estándares.

\section{REFERENCIAS BIBLIOGRÁFICAS}

Buendía, L. y López, R. (1999). Concepciones de los profesores de educación secundaria sobreevaluación. Educación XXI. Revista de la Facultad de Educación, 125 - 153.

Cevallos, D. (2014). La Calidad Educativa en la realidad Universitaria Peruana frente al Contexto Latinoamericano. Revista de la Universidad Católica Santo Toribio de Mogrovejo Chiclayo, 7(1), 3-8.

Constitución Política del Perú [Const]. Décimo Tercera Edición Oficial Texto actualizado con las reformas ratificadas en el Referéndum de 2018. (Perú). 
Decreto Supremo N 016-2015-MINEDU. Por la cual aprueban la Política de Aseguramiento dela Calidad de la Educación Superior Universitaria. 26 de setiembre de 2015.

International Organization for Standardization - ISO. (2015). ISO 9000:2015: sistemas de gestión de la calidad: fundamentos y vocabulario. Numeral 3.3.2.

https://www.iso.org/obp/ui/\#iso:std:iso:9000:ed-4:v1:es

Resolución de Consejo Directivo N 006-2015-SUNEDU/CD. Por la cual aprueban el Modelo de Licenciamiento y su implementación en el Sistema Universitario Peruano. 24 de noviembre de 2015.

Sovero, H. (2008). Gestión educacional. Gestión pedagógica. (Vol. 3). Palomino E.I.R.L. Suarez, R. La Educación. Estrategias De Enseñanza-Aprendizaje.

Yamada, G. y Castro, J. (2013). Calidad y acreditación de le educación superior: retos urgentes para el Perú. Universidad del Pacífico.

Yamada, G. Castro, J. y Rivera, M. (2013). Educación superior en el Perú: Retos para el aseguramiento de la calidad. SINEACE.

Modelo de Acreditación para Programas de Estudios de Educación Superior Universitaria - SINEAC. (2016).

Modelo de Acreditación para Programas de Estudios de Educación Superior Universitaria Explicación de estándares y criterios. SINEACE. (2017).

Toro, J. (2012). Gestión interna de la calidad en las instituciones de educación superior. Ril; Cinda. 